\title{
Efficient Coding of Spatial Information in the Primate Retina
}

\author{
Eizaburo Doi, ${ }^{1,3}$ Jeffrey L. Gauthier, ${ }^{3}$ Greg D. Field, ${ }^{3}$ Jonathon Shlens, ${ }^{1,2,3}$ Alexander Sher, ${ }^{4}$ Martin Greschner, ${ }^{3}$ \\ Timothy A. Machado, ${ }^{3}$ Lauren H. Jepson, ${ }^{3}$ Keith Mathieson, ${ }^{5}$ Deborah E. Gunning, ${ }^{5}$ Alan M. Litke, ${ }^{4}$ Liam Paninski, ${ }^{6}$ \\ E. J. Chichilnisky, ${ }^{3 *}$ and Eero P. Simoncelli ${ }^{1,2 *}$ \\ ${ }^{1}$ Center for Neural Science and ${ }^{2}$ Howard Hughes Medical Institute, New York University, New York, New York 10003, ${ }^{3}$ Systems Neurobiology Laboratories, \\ The Salk Institute, La Jolla, California 92037, ${ }^{4}$ Santa Cruz Institute for Particle Physics, University of California, Santa Cruz, Santa Cruz, California 95064, \\ ${ }^{5}$ Department of Physics and Astronomy, University of Glasgow, Glasgow G12 8QQ, United Kingdom, and ${ }^{6}$ Department of Statistics and Center for \\ Theoretical Neuroscience, Columbia University, New York, New York 10027
}

Sensory neurons have been hypothesized to efficiently encode signals from the natural environment subject to resource constraints. The predictions of this efficient coding hypothesis regarding the spatial filtering properties of the visual system have been found consistent with human perception, but they have not been compared directly with neural responses. Here, we analyze the information that retinal ganglion cells transmit to the brain about the spatial information in natural images subject to three resource constraints: the number of retinal ganglion cells, their total response variances, and their total synaptic strengths. We derive a model that optimizes the transmitted information and compare it directly with measurements of complete functional connectivity between cone photoreceptors and the four major types of ganglion cells in the primate retina, obtained at single-cell resolution. We find that the ganglion cell population exhibited $80 \%$ efficiency in transmitting spatial information relative to the model. Both the retina and the model exhibited high redundancy $(\sim 30 \%)$ among ganglion cells of the same cell type. A novel and unique prediction of efficient coding, the relationships between projection patterns of individual cones to all ganglion cells, was consistent with the observed projection patterns in the retina. These results indicate a high level of efficiency with near-optimal redundancy in visual signaling by the retina.

\section{Introduction}

The computations performed by neural circuits are essential for survival but come at a cost. It has been hypothesized that the early stages of sensory processing have evolved to accurately encode environmental signals with the minimal consumption of biological resources (Attneave, 1954; Barlow, 1961; Atick and Redlich, 1990; van Hateren, 1992b; Laughlin, 2001; Chklovskii et al., 2002; Bialek et al., 2006). This theoretical hypothesis, generally known as efficient coding, has been used to explain a variety of observed properties of sensory systems (Laughlin, 1981; Srinivasan et al., 1982; Atick and Redlich, 1992; van Hateren, 1992a; Rieke et al., 1995; Dan et al., 1996; Olshausen and Field, 1996; Baddeley et al., 1997; Bell and Sejnowski, 1997; Machens et al., 2001;

\footnotetext{
Received Aug. 23, 2012; accepted Aug. 25, 2012.

Author contributions: E.D., E.J.C., and E.P.S. designed research; E.D. performed research; E.D., J.L.G., G.D.F., J.S., A.S., M.G., T.A.M., L.H.J., K.M., D.E.G., A.M.L., L.P., E.J.C., and E.P.S. contributed unpublished reagents/analytic tools; E.D., J.L.G., and G.D.F. analyzed data; E.D., E.J.C., and E.P.S. wrote the paper.

*E.J.C. and E.P.S. contributed equally to this work.

This work was supported by National Institutes of Health Grant EY018003 (L.P., E.J.C., and E.P.S.) and Howard Hughes Medical Institute (E.P.S.).

The authors declare no competing financial interests.

This article is freely available online through the JNeurosci Open Choice option.

Correspondence should be addressed to Eizaburo Doi, Glennan Room 321, 10900 Euclid Avenue, Cleveland, OH 44106. E-mail: edoi@case.edu.

E. Doi's present address: Department of Electrical Engineering and Computer Science, Case Western Reserve University, Cleveland, $\mathrm{OH} 44106$.

J. L. Gauthier's present address: Princeton Neuroscience Institute, Princeton University, Princeton, NJ 08544.

G. D. Field's present address: Keck School of Medicine, University of Southern California, Los Angeles, CA 90089.

T. A. Machado's present address: Center for Theoretical Neuroscience, Columbia University, New York, NY 10027.

DOI:10.1523/JNEUROSCI.4036-12.2012

Copyright $\odot 2012$ the authors $\quad 0270-6474 / 12 / 3216256-09 \$ 15.00 / 0$
}

Schwartz and Simoncelli, 2001; Vincent and Baddeley, 2003; Chechik et al., 2006; Graham et al., 2006; Smith and Lewicki, 2006; Doi and Lewicki, 2007; Borghuis et al., 2008; Liu et al., 2009).

The retina provides a natural choice for the study of coding efficiency, given its role in transmitting visual information to the brain and the extensive literature documenting its anatomical and functional properties. Previous work showed that behavioral measurements of bandpass contrast sensitivity in the primate visual system (Kelly, 1972; De Valois et al., 1974) are generally consistent with efficient coding (Atick and Redlich, 1992; van Hateren, 1992b; Dan et al., 1996). However, it is still far from clear whether the specific organization of the retinal circuitryconsisting of distinct types of retinal ganglion cells (RGCs) (Masland, 2001), each blanketing the entire visual field with a lattice of irregularly shaped receptive fields (Gauthier et al., 2009) - is consistent with efficient coding. Although the patterns of spike trains observed in individual retinal neurons appear to reflect metabolically efficient information transmission (Balasubramanian and Berry, 2002; Koch et al., 2004), recent studies have shown significant redundancy between pairs of retinal responses (Meister et al., 1995; Puchalla et al., 2005; Schneidman et al., 2006; Shlens et al., 2006; Ala-Laurila et al., 2011), potentially at odds with coding efficiency (Puchalla et al., 2005; Ala-Laurila et al., 2011).

In this paper, we test the efficiency of spatial processing of visual signals that transforms the responses of cone photoreceptors to those of RGCs. Using data from a high-density multielectrode array, we measure the functional connectivity between the lattice of cones and multiple complete populations of RGCs. We then compare these with the proposed connectivity model opti- 

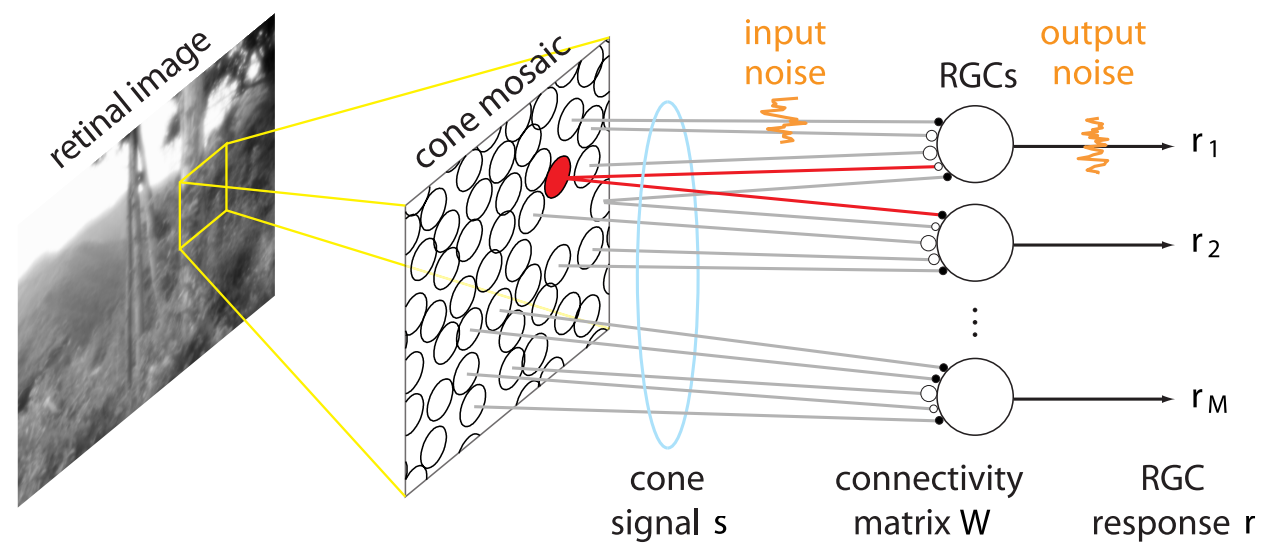

Figure 1. Functional model of RGC responses, used for assessment of efficient coding theory. The model consists of retinal images of natural scenes, represented in the cone photoreceptor mosaic obtained from the data. A linear combination of these cone signals, specified by a connectivity matrix, W, governs model RGC responses. White Gaussian noise is added before and after the linear combination, with amplitude set in accordance with previous studies (Atick and Redlich, 1990; van Hateren, 1993). The set of connectivity weights arising from a single cone (red) constitute the projective field (PF) of that cone.

mized for transmitting spatial information in natural images subject to the same neural resources found in the measured retinal region. The results indicate high efficiency of the retinal circuitry and an accompanying redundancy of neural signals conveyed to the brain.

\section{Materials and Methods}

Physiological data. We examined electrophysiological recordings obtained from three macaque monkeys based on segments of retina taken from regions at 27,38 , and 28 degrees of eccentricity from the fovea, respectively. Stimulus generation and calibration, spike identification, cell-type classification, and estimation of functional connectivity have been described by Field et al. (2010). Briefly, the connectivities were obtained by reverse correlating the measured spike trains of RGCs against the stimuli, which were spatiotemporal white noise with red, green, and blue monitor primary intensities drawn from a binary distribution. Pixel sizes (with side length $\sim 1.5 \mathrm{~min}$ of arc) were small enough that the spike-triggered average revealed the locations of individual cones as well as their identity: (L)ong, (M)edium, or (S)hort wavelength sensitive. The measurements of functional connectivity were restricted to $\mathrm{L}$ and $\mathrm{M}$ cones and to ON-Parasol, OFF-Parasol, ON-Midget, and OFFMidget RGCs, identified by the spatiotemporal properties of their receptive fields. Each type tiled the region of retina examined, in all three datasets. The numbers of cones and RGCs in the three datasets were, respectively, $\{706,131\},\{520,89\}$, and $\{569,92\}$, corresponding to a cell ratio of $5.8 \pm 0.4$ (mean $\pm \mathrm{SD})$. The ratios broken down by RGC type were $71.5 \pm 6.7$ (ON-Parasol), $70.2 \pm 9.6$ (OFF-Parasol), $13.7 \pm 0.5$ (ON-Midget), and $14.1 \pm 1.7$ (OFF-Midget). The results shown in Figures $2-5$ were obtained with the first dataset. Consistent results were obtained with the other two datasets.

$R G C$ response model. For analysis of coding efficiency, we assume the following functional model of RGC responses:

$$
\mathbf{r}=\mathbf{W}(\mathbf{s}+\boldsymbol{\nu})+\boldsymbol{\delta},
$$

where $\mathbf{s}$ is an $\mathrm{N}$-dimensional vector of cone responses, $\nu$ (input noise) is Gaussian white noise with variance $\sigma_{v}^{2}, \mathrm{~W}$ is an $M \times N$ matrix expressing the functional connectivity between cones and RGCs, and $\delta$ (output noise) is Gaussian white noise with variance $\sigma_{\delta}^{2}$. The resulting $M$-dimensional vector, $\mathbf{r}$, represents the response of the RGCs. The model structure is similar to that of previous studies (Linsker, 1989; Atick and Redlich, 1990; Atick et al., 1990; Bialek et al., 1991; van Hateren, 1992b) but does not assume a regular lattice of cones. The connectivity matrix $\mathbf{W}$ is permitted to represent an inhomogeneous RGC population of arbitrary size.

The information transmitted by the RGC population was estimated by assuming a Gaussian probability model for the cone signal $\mathbf{s}$. The empir- ical covariance of the cone responses, $\mathrm{C}_{\mathrm{s}}$, was computed as follows. First, a set of 62 calibrated achromatic natural images (Doi et al., 2003) was blurred (Fig. 1) according to the modulation transfer function of the human eye (Navarro et al., 1993) at 30, 40, and 30 degrees of eccentricity for the three retina datasets. Next, the retinal images were sampled using the physiologically measured cone mosaic, simulating photon absorption values across the cone lattice. These were transformed with a compressive cone nonlinearity followed by subtraction of the mean across stimuli (Baylor et al., 1987; Doi et al., 2003). For accurate covariance estimation, cone signals were sampled from 6,200,000 randomly selected image patches.

The model also includes input noise [capturing the effects of photon shot noise, phototransduction noise, and membrane noise in the cone (Srinivasan et al., 1982; Atick and Redlich, 1990; van Hateren, 1993; Ruderman, 1994)] and output noise [capturing noise introduced after the linear combination of cone responses, including synaptic noise, RGC membrane noise, and the loss of information in the conversion of synaptic currents to spikes (Srinivasan et al., 1982; Atick and Redlich, 1990; van Hateren, 1993; Ruderman, 1994; Dhingra and Smith, 2004)]. The noise variances, $\sigma_{\nu}^{2}$ and $\sigma_{\delta}^{2}$, were selected to produce signal-to-noise ratios (SNRs) of 1 and 10 (corresponding to 0 and $10 \mathrm{~dB}$ ), respectively. The input SNR was defined as $\Sigma_{j=1}^{N} \operatorname{Var}\left(s_{j}\right) / N \sigma_{v}^{2}$, where $N$ is the number of cones and $s_{j}$ is the $j$ th cone signal, and the output SNR was defined similarly as $\operatorname{tr}\left(\mathbf{W C}_{\mathbf{s}} \mathbf{W}^{T}+\sigma_{\nu}^{2} \mathbf{W} \mathbf{W}^{T}\right) / M \sigma_{\delta}^{2}$ [note that the numerator is the sum of variances of RGC responses before output noise is added, $\mathbf{W}(\mathbf{s}+$ $\nu)]$ ). These choices are not strongly constrained by currently available measurements. However, perturbing these SNR values by $\pm 10 \mathrm{~dB}$ produced minor changes in the results.

Given the linear-Gaussian RGC response model, the mutual information between cone signal $\mathbf{s}$ and the RGC response $\mathbf{r}$ can be computed explicitly for any given connectivity matrix W (Atick and Redlich, 1990; Atick et al., 1990; van Hateren, 1992b; Campa et al., 1995):

$$
I(\mathbf{s} ; \mathbf{r})=\frac{1}{2} \log _{2} \frac{\operatorname{det}\left(\mathbf{W C}_{\mathbf{s}} \mathbf{W}^{T}+\sigma_{\nu}^{2} \mathbf{W} \mathbf{W}^{T}+\sigma_{\delta}^{2} \mathbf{I}\right)}{\operatorname{det}\left(\sigma_{\nu}^{2} \mathbf{W} \mathbf{W}^{T}+\sigma_{\delta}^{2} \mathbf{I}\right)},
$$

where $\mathbf{I}$ is the identity matrix.

The information present in the cone responses is computed as the mutual information between the cone signal, $\mathrm{s}$, and the noise-corrupted cone responses, $\mathbf{s}+\nu$ :

$$
I(\mathbf{s} ; \mathbf{r})=\frac{1}{2} \log _{2} \frac{\operatorname{det}\left(\mathbf{C}_{\mathbf{s}}+\sigma_{\nu}^{2} \mathbf{I}\right)}{\operatorname{det}\left(\sigma_{\nu}^{2} \mathbf{I}\right)},
$$

Efficient coding solution. The connectivity matrix $\mathbf{W}$ that maximizes the transmitted information (Eq. 2) was derived subject to three constraints. First, the size of $\mathbf{W}$ was chosen to match that of the physiological connec- 
tivity matrix $\mathbf{W}_{\text {ret }}$ (i.e., numbers of cones and RGCs). Second, the total response variance,

$$
\rho(\mathbf{W})=\sum_{i=1}^{M} \operatorname{Var}\left(r_{i}\right)=\operatorname{tr}\left(\mathbf{W C}_{\mathbf{s}} \mathbf{W}^{T}+\sigma_{\nu}^{2} \mathbf{W} \mathbf{W}^{T}+\sigma_{\delta}^{2} \mathbf{I}\right) .
$$

was constrained to match that of $\mathbf{W}_{\text {ret }}$. Third, the total squared synaptic strength,

$$
\tau(\mathbf{W})=\sum_{i=1}^{M} \sum_{j=1}^{N} W_{i j}^{2}=\operatorname{tr}\left(\mathbf{W} \mathbf{W}^{T}\right),
$$

was constrained to match that of $\mathbf{W}_{\text {ret }}$. Although each of these constraints may be found in previous literature, the present model is the first to include all three. In particular, most previous studies (Atick and Redlich, 1990; Atick et al., 1990; van Hateren, 1992b; Haft and van Hemmen, 1998) assumed only the total response variance constraint without matching cell numbers to physiological data, and Campa et al. (1995) provided the analysis for arbitrary cell numbers but convergent cell ratio $(M \leq N)$. Our analysis is more general in that the cell ratio may also be divergent $(M>N)$ and in the inclusion of two additional constraints. We have shown that those constraints play an important role in shaping the solution (Doi et al., 2010).

The optimal connectivity is computed by solving a constrained optimization problem for $\mathbf{W}$ that maximizes Equation 2 subject to the two equality constraints (Eqs. 4, 5). The conventional procedure of rewriting the objective function using Lagrange multiplier terms for the two equality constraints was adopted (Chong and Zak, 2001). The resulting problem is not easily solved, because the primary objective function (Eq. 2) is not convex with respect to $\mathbf{W}$. The following analysis transforms the problem into a convex one, thus guaranteeing a globally optimal solution for $\mathbf{W}$.

The connectivity matrix can be reexpressed as $\mathbf{W}=\mathbf{P} \Omega \mathbf{Q}^{T}$, using the singular-value decomposition (Strang, 2005), in which the first and third matrices are orthogonal and the middle one diagonal. First, the first orthogonal matrix, $\mathbf{P}$, does not affect the values of either the objective function (Eq. 2) or the two constraints (Eqs. 4, 5) and thus can be chosen arbitrarily (see below, Best-fitting solutions). Second, it can be shown that, for the optimal connectivity, the second orthogonal matrix, Q, should be set to the eigenvector matrix of the signal covariance matrix (Campa et al., 1995). This implies that the signal is first represented in the coordinates of its principal axes, as in principal component analysis (Zhaoping, 2006). Once represented in this coordinate system, the signal is modulated along the axes (via the diagonal matrix $\Omega$ ) and finally represented with the new basis functions (columns of $\mathbf{P}$ ) with dimension equal to the number of RGCs. What remains is to optimize the diagonal entries of $\Omega$, denoted as $\omega_{i}$. Thus, the objective function (Eq. 2) is now reduced to a concave function with respect to the squares of those diagonal entries:

$$
I(\mathbf{s} ; \mathbf{r})=\frac{1}{2} \sum_{i=1}^{\min \{M, N\}} \log _{2}\left(\frac{\lambda_{i}}{\sigma_{\nu}^{2}+\sigma_{\delta}^{2} / \omega_{i}^{2}}+1\right) .
$$

One can show that the second derivative of Equation 6 with respect to $\omega_{i}^{2}$ is always strictly negative. It is useful to note that $\frac{\lambda_{i}}{\sigma_{v}^{2}+\sigma_{\delta}^{2} / \omega_{i}^{2}}=\mathrm{SNR}_{i}$ represents the effective SNR of the ith signal eigenvalue in the neural representation (Rieke et al., 1997), and Equation 6 is the sum of information over noisy Gaussian channels, $1 / 2 \log _{2}\left(\mathrm{SNR}_{i}+1\right)$ (Cover and Thomas, 2006). The two constraints (Eqs. 4, 5) are also reduced, respectively, to

$$
\rho(\mathbf{W})=\rho\left(\left\{\omega_{i}^{2}\right\}_{i=1}^{\min }\{M, N\}\right)=\sum_{i=1}^{\min \{M, N\}} \omega_{i}^{2}\left(\lambda_{i}^{2}+\sigma_{\nu}^{2}\right),
$$

where $\lambda_{i}^{2}$ are the eigenvalues of $\mathbf{C}_{\boldsymbol{s}}$, and

$$
\tau(\mathbf{W})=\tau\left(\left\{\omega_{i}^{2}\right\}_{i=1}^{\min \{M, N\}}\right)=\sum_{i=1}^{\min \{M, N\}} \omega_{i}^{2} .
$$

The feasible set of optimization parameters, $\omega_{i}^{2}$, is convex. Because the objective function is concave and the feasible set is convex, this optimization problem can be solved using the Karush-Kuhn-Tucker condition, a standard result in optimization theory (Chong and Zak, 2001).

It is important to note that the efficient coding solution, $\mathbf{W}_{\mathrm{op}}$, is not a whitening matrix except for the special case in which the input noise is zero and the resources are solely constrained by the total response variance. Because the input noise of the retina is significant (Ala-Laurila et al., 2011) and the synaptic weights in the retina are naturally assumed to have a direct bearing on the cost of synaptic resource usage, $\mathbf{W}_{\text {opt }}$ will never be a whitening matrix for the retinal transform.

Best-fitting solution. A set of connectivity matrices that are equally optimal is given by $\mathbf{P} \mathbf{W}_{\text {opt }}$, where $\mathbf{P}$ is an orthogonal matrix, and $\mathbf{W}_{\text {opt }}$ is an arbitrarily selected optimal connectivity matrix. We obtained $\mathbf{W}_{\mathrm{opt}}$ with $\mathbf{P}_{\text {rnd }} \boldsymbol{\Omega}_{\text {opt }} \mathbf{Q}_{\text {opt }}^{T}$, where $\mathbf{P}_{\text {rnd }}$ is a left orthogonal matrix of the singular value decomposition of an $M$-dimensional matrix with elements randomly drawn from the normal distribution, and $\Omega_{\text {opt }}$ and $\mathbf{Q}_{\text {opt }}$ are the optimal components of $\mathbf{W}$ as defined in the previous section. The bestfitting orthogonal matrix for the optimal connectivity matrix, $\mathbf{P}_{\text {fit }}$, is given by the minimizer of the squared error, $\varepsilon(\mathbf{P})=\left\|\mathbf{P W}_{\mathrm{opt}}-\mathbf{W}_{\text {ret }}\right\|_{F}^{2}$, where $\|. .\|_{F}$ denotes Frobenius norm (the sum of squares of the matrix entries). This type of optimization is known as the orthogonal Procrustes problem and can be solved in closed form (Gower and Dijksterhuis, 2004). We reported the squared error relative to the data variance, $\varepsilon /\left\|\mathbf{W}_{\text {ret }}\right\|_{F}^{2}$.

Each RGC receptive field outlined in Figure 2 is the effective linear weighting that maps visual stimuli to RGC response. This is constructed by convolving the receptive fields of individual cones (depicted by small circles in Fig. 1) with the point spread function of the eye at the relevant eccentricity (Navarro et al., 1993) and then summing all these cone profiles with the weights specified by the connection matrix entries for that RGC.

Boundary handling. Most of the analyses were conducted without special handling of the boundaries of the recording. Exceptions are as follows. In Figure 2, those cones on the boundary of the retinal patch were excluded. To solve for $\mathbf{P}_{\text {fit }}$, several RGCs on the boundary of the retinal patch were excluded. In this case, $\mathbf{P}_{\text {fit }}$ is rectangular with the row vectors orthogonal to each other, and a standard solution for the orthogonal Procrustes problem cannot be used (Gower and Dijksterhuis, 2004). Thus, $\mathbf{P}_{\text {fit }}$ was obtained numerically by iterating the gradient descent to minimize the squared error and the orthogonalization of rows of $\mathbf{P}_{\text {fit }}$. The analysis was repeated without this boundary handling, producing similar but noisier results.

Unique prediction about connectivity. The family of efficient connectivity matrices $\mathbf{W}_{\text {opt }}$, which differ from each other only by an orthogonal transformation, provides a novel theoretical prediction that can be compared with data: the uniquely specified matrix $\mathbf{Z}=\mathbf{W}_{\mathrm{opt}}^{T} \mathbf{W}_{\mathrm{opt}}$, for any given $\mathbf{W}_{\text {opt }}$. This matrix is a unique prediction of efficient coding, because it is determined solely by the two unique optimal components, $\Omega_{\mathrm{opt}}$ and $\mathbf{Q}_{\mathrm{opt}}$, and is invariant to the choice of the orthogonal matrix $\mathbf{P}$ [because $\left(\mathbf{P} \mathbf{W}_{\text {opt }}\right)^{T}\left(\mathbf{P W}_{\text {opt }}\right)=\mathbf{W}_{\text {opt }}^{T} \mathbf{P}^{T} \mathbf{P} \mathbf{W}_{\text {opt }}=\mathbf{W}_{\text {opt }}^{T} \mathbf{W}_{\text {opt }}$, for any orthogonal $\mathbf{P}$ ]. The individual elements $Z_{i j}$ of $\mathbf{Z}$ represent the inner product of the $i$ th and $j$ th columns of the connectivity matrix, which contain the weights of the $i$ th and $j$ th cone projective fields (PFs). If $i=j$, then $Z_{i j}$ indicates the squared strength (or norm) of the PF of the $i$ th cone.

Definition of redundancy. A general form of redundancy that quantifies the informational overlap in a neural population is the sum of the information transmitted by disjoint subpopulations of neurons (e.g., individual neurons), $\mathbf{r}_{i}$, minus the information transmitted jointly by the population formed from their union, $\left\{\mathbf{r}_{i} ; \mathrm{i}=1, \ldots, M\right\}$,

$$
\Delta I\left(\left\{\mathbf{r}_{i} ; i=1, \ldots, M\right\}\right)=\sum_{i=1}^{M} I\left(\mathbf{s} ; \mathbf{r}_{i}\right)-I\left(\mathbf{s} ;\left\{\mathbf{r}_{i} ; i=1, \ldots, M\right\}\right)
$$

The negative of this quantity is also referred to as synergy (Gawne and Richmond, 1993; Brenner et al., 2000; Machens et al., 2001; Schneidman et al., 2003; Latham and Nirenberg, 2005). 
(a)

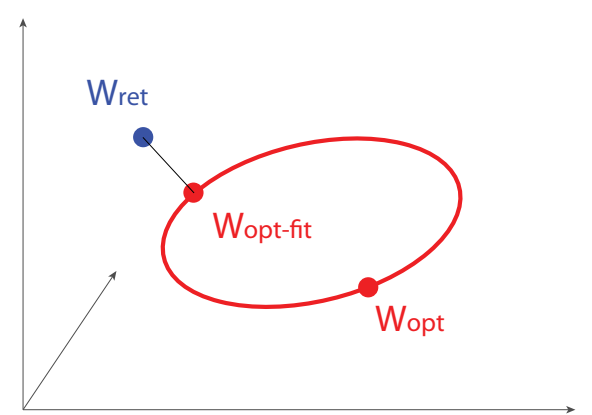

high-dimensional W space

(c) Optimal (best-fit): Wopt-fit

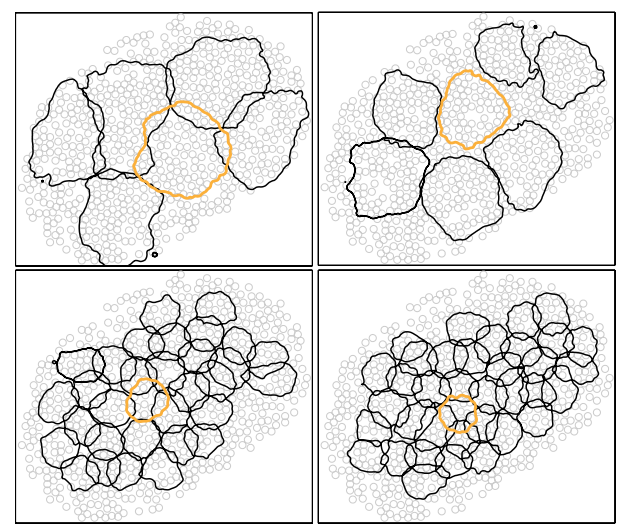

(b) Retinal: Wret

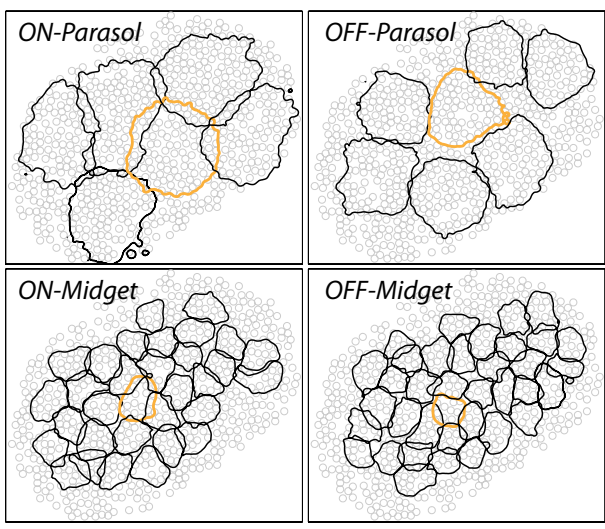

(d) Optimal (arbitrary): Wopt

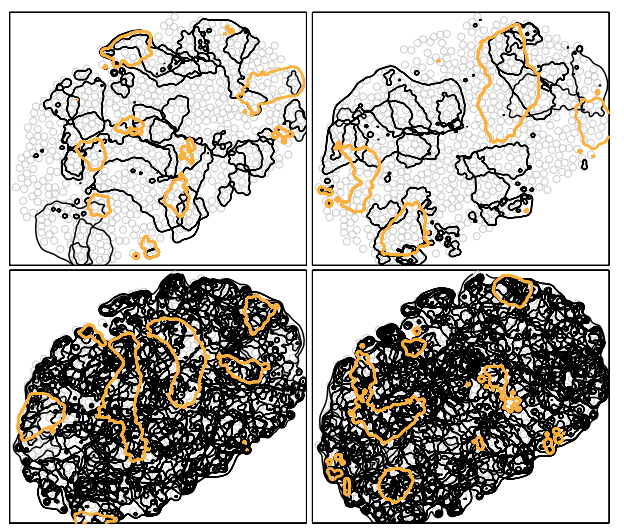

Figure 2. Comparison of RGC receptive field spatial structure to theoretical predictions. $\boldsymbol{a}$, A schematic of connectivity matrices. Each point in space corresponds to one connectivity matrix, and three such matrices are indicated. Note that $\mathbf{P W}_{\text {opt }}$ is the optimal connectivity with any choice of orthogonal matrix $\mathbf{P}$, constituting a manifold of the optimal solution as illustrated by the ellipsoid. $\boldsymbol{b}$, Measured RGC receptive fields, i.e., (the rows of) $\mathbf{W}_{\text {ret }} \boldsymbol{c}$, The optimal connectivity closest to the retinal data, $\mathbf{W}_{\text {opt-fit }} \boldsymbol{d}$, Arbitrarily chosen optimal connectivity, $\mathbf{W}_{\text {opt }}$. Each panel in $\boldsymbol{b}-\boldsymbol{d}$ shows contours of receptive fields of the four major RGC types at $30 \%$ of maximum, superimposed on the cone lattice. A contour from one cell per each panel is highlighted in orange for clarity.

The portion of information that is uniquely conveyed by the $k$ th neuron is given by

$$
I_{\text {uni }}(k)=I(\mathbf{s} ; \mathbf{r})-I\left(\mathbf{s} ; \mathbf{r}_{\neg k}\right),
$$

where $\mathbf{r}$ is the responses of the full neural population, and $\mathbf{r}_{\neg k}$ is the responses of the same population, with the $k$ th neuron removed. The portion of information conveyed by the $k$ th neuron that is also conveyed by all the other neurons in the population is thus given by

$$
\begin{aligned}
\Delta I_{\mathrm{sc}}(k) & =I\left(\mathbf{s} ; r_{k}\right)-I_{\text {uni }}(k) \\
& =I\left(\mathbf{s} ; r_{k}\right)+I\left(\mathbf{s} ; \mathbf{r}_{\neg k}\right)-I(\mathbf{s} ; r),
\end{aligned}
$$

referred to here as the single-cell redundancy. Note that this is a special case of Equation 9 because the union of $r_{k}$ and $\mathbf{r}_{\neg k}$ is $\mathbf{r}$. In Results, the ratio of single-cell redundancy to the information conveyed by the single neuron is reported, $\Delta I_{\mathrm{sc}}(k) / I\left(\mathbf{s} ; r_{k}\right)$.

The pairwise redundancy shown in Figure 4 is also given by Equation 9 , with the population consisting of two neurons. The quantity reported in Figure 4 is normalized in accordance with previous work (Puchalla et al., 2005):

$$
\frac{I\left(\mathbf{s} ; r_{1}\right)+I\left(\mathbf{s} ; r_{2}\right)-I\left(\mathbf{s} ;\left\{r_{1}, r_{2}\right\}\right)}{\min \left\{I\left(\mathbf{s} ; r_{1}\right), I\left(\mathbf{s} ; r_{2}\right)\right\}}
$$

for which the maximum possible value, corresponding to a completely redundant pair, is 1 (Machens et al., 2001).

To gain insights into efficient coding, it is also useful to examine the redundancy of Equation 9 for the full set of individual neurons within a population. The information transmitted by the entire population can be expressed as the sum of information transmitted by individual neurons, minus the redundancy:

$$
I(\mathbf{s} ; \mathbf{r})=\sum_{i=1}^{\mathrm{M}} I\left(\mathbf{s} ; r_{i}\right)-\Delta I(\mathbf{r}) .
$$

This implies that maximizing information, $I(\mathbf{s} ; \mathbf{r})$, is a tradeoff between maximizing the sum of transmitted information by individual neurons (the first term on the right side) and minimizing the redundancies between them (the second term). This tradeoff has been discussed previously with a different definition of redundancy (Borghuis et al., 2008; Balasubramanian and Sterling, 2009). Note that Equation 12 makes it explicit that redundancy reduction is not equivalent to information maximization.

Simple developmental model of retinal connectivity. We simulated a developmental model (see Fig. 5) to obtain an alternative connectivity matrix, W. The elements of this matrix, $W_{i j}$, were adjusted using an iterative learning rule with initial conditions. This iteration was implemented to achieve two goals.

(1) Response variance, $\sigma^{2}$, should equal the average variance of RGCs with the connectivity $\mathbf{W}_{\text {ret }}$ in response to natural images. The target variance was set for each individual RGC type separately. In each iteration, the value $W_{i j}$ (connectivity from the $j$ th cone to the $i$ th model RGC) was incremented by a local update rule:

$$
\Delta W_{i j} \propto-\left(\sigma_{i}^{2}-\sigma^{2}\right)\left\langle\left(s_{j}+\nu_{j}\right) u_{i}\right\rangle,
$$

where $\sigma_{i}^{2}$ is the response variance of the $i$ th neuron, $s_{j}$ and $\nu_{j}$ are, respectively, the signal and noise of the $j$ th cone, $u_{i}$ is the response of the $i$ th 
neuron, and $\langle\ldots\rangle$ indicates the ensemble average over the presentation of natural images.

(2) Magnitude of the cone PF within each RGC type, $\phi$, should equal the average magnitude of the PF in the measured connectivity $\mathbf{W}_{\text {ret. }}$. A constant magnitude of cone PF per RGC type ensures that each RGC type uniformly samples the cone lattice without gaps, tiling the region of retina (Gauthier et al., 2009). In each iteration, $W_{i j}$ was incremented by another local update rule:

$$
\Delta W_{i j} \propto-\left(\phi_{j}^{2}-\phi^{2}\right) W_{i j}
$$

where $\phi_{j}$ is the PF norm of the $j$ th cone . Importantly, the optimal connectivity exhibits nearly constant PF norm for an entire RGC population (and we proved that this is exactly constant in the ideal case of a regular cone lattice with shift-invariant natural images). Hence, this biologically plausible rule leads to connectivity that satisfies a necessary condition for optimal information transmission.

The connectivity matrix was initialized by the model receptive fields (rows of $\mathbf{W}$ ) with spatially localized Gaussian profiles with center locations taken from the data and SD equal to half the distance to the nearest RGC of the same type (Devries and Baylor, 1997). This effectively prohibited long-range connections, because the objective function is nonconvex (fourth power of $W_{i j}$ ) and has local minima. Iterative adjustment of the entries of $\mathbf{W}$ terminated when both target values in goals 1 and 2 were achieved simultaneously. These conditions satisfy the constraints of total response variance and squared synaptic strength, respectively, and hence allowed a fair comparison of the resulting connectivity with retinal $\left(\mathbf{W}_{\text {ret }}\right)$ and efficient $\left(\mathbf{W}_{\text {opt }}\right)$ connectivity matrices.

\section{Results}

The circuitry of the retina transforms the visual information captured by a cone photoreceptor mosaic into the electrical signals in multiple types of RGCs, which is then transmitted to the brain. We compared the spatial properties of a linear approximation of this transformation, measured at single-cell resolution, against predictions of efficient coding theory.

\section{Measuring and modeling spatial processing in the retina}

The spatial transformation from cone to RGC responses was measured using multielectrode recordings of peripheral macaque monkey retina ex vivo (Field et al., 2010). These recordings sampled the electrical activity of complete populations of the four numerically dominant primate RGC types: ON-Parasol, OFF-Parasol, ONMidget, and OFF-Midget. Fine-grained visual stimulation was used to measure the spatial receptive fields of complete populations of these RGCs at the resolution of individual cones. These measurements quantified the strength of functional connection from every cone to every recorded RGC over a region of the retina.

The predictions of efficient coding were derived using a simplified response model, constructed to be comparable with the data while incorporating the statistical properties of natural images, noise, and biological constraints (Fig. 1). Achromatic natural images were obtained from a database (Doi et al., 2003), blurred according to the optics of the eye (Navarro et al., 1993), and represented in terms of the elicited photon absorptions of cones laid out in an irregular lattice as measured using physiological data (Field et al., 2010). These model cone signals, transformed by an instantaneous compressive nonlinearity (Baylor et al., 1987; Doi et al., 2003) and corrupted by noise, were combined linearly to produce model RGC signals. Finally, model RGC signals were corrupted with additive independent noise. The free parameters of the model were the strengths of inputs from all the model cones to all the model RGCs, summarized in a connectivity matrix $\mathbf{W}$. By construction, $\mathbf{W}$ is directly comparable with the physiologically measured weights of cone inputs to RGCs, $\mathbf{W}_{\text {ret }}$. To test the predictions of efficient coding for the retinal circuitry,
$\mathbf{W}_{\text {ret }}$ was compared with an optimal connectivity matrix, $\mathbf{W}_{\text {opt }}$, that was numerically optimized for information transmission. This optimization was performed subject to three resource constraints relevant to the retinal circuitry (see Materials and Methods): (1) number of RGCs (Campa et al., 1995; Doi and Lewicki, 2007); (2) total response variance of RGCs (Atick and Redlich, 1990, 1992; Atick et al., 1990; van Hateren, 1992b, 1993; Ruderman, 1994; Haft and van Hemmen, 1998; Doi and Lewicki, 2007); and (3) total squared synaptic strengths (Campa et al., 1995).

\section{Coding efficiency of the retina}

How efficiently does the retina process the spatial information in natural images? To answer this question, information transmission was calculated for two different model RGC populations: one with the physiologically measured connectivity $\left(\mathbf{W}_{\text {ret }}\right)$ and the other with optimal connectivity subject to resource constraints $\left(\mathbf{W}_{\text {opt }}\right)$. Comparison of these values indicates the degree to which the retinal connectivity is efficient. In three recordings from different retinas, the retinal connectivity preserved $59.4 \pm$ $8.1 \%$ (mean $\pm \mathrm{SD}$ across datasets) of the visual information present in the cone lattice (defined by the information about cone signal that is gained after input noise is added; see Materials and Methods) compared with $74.8 \pm 6.7 \%$ preserved with the optimal connectivity. Thus, RGCs transmit a large fraction of the visual information possible, exhibiting an overall efficiency of $\sim 80 \%$ of the maximum possible $(82.4,81.2$, and $74.2 \%$, respectively, for three datasets).

\section{Receptive field organization}

Does the retinal circuitry exhibit spatial structure consistent with efficient coding? A direct comparison of the measured RGC receptive fields (rows of $\mathbf{W}_{\text {ret }}$ ) with the optimal receptive fields (rows of $\mathbf{W}_{\text {opt }}$ ) is not informative, because the optimal connectivity matrix $\mathbf{W}_{\text {opt }}$ is not uniquely specified by efficient coding. Specifically, multiplying a connectivity matrix by any orthogonal matrix $\mathbf{P}$ yields a new connectivity matrix that uses the same resources and transmits the same amount of information (see Materials and Methods). Thus, spatial receptive field structure does not provide a unique test of efficient coding.

A partial test of efficiency can be developed by finding, within the family of optimal connectivity matrices $\mathbf{W}_{\text {opt }}$, the single connectivity matrix $\mathbf{W}_{\text {opt-fit }}$ that most closely matches the data (Fig. $2 a$ ). Mathematically, finding this matrix is equivalent to starting with any choice of $\mathbf{W}_{\text {opt }}$ and finding an orthogonal matrix $\mathbf{P}$ that minimizes $\left\|\mathbf{W}_{\text {ret }}-\mathbf{P} \mathbf{W}_{\text {opt }}\right\|^{2}$. A close match between $\mathbf{W}_{\text {ret }}$ and $\mathbf{W}_{\text {opt-fit }}$ would indicate efficient spatial structure of retinal receptive fields. The results show that indeed the receptive fields in $\mathbf{W}_{\text {opt-fit }}$ are similar to those in the retina (Fig. $2 b, c$ ). In three datasets, the squared error of $\mathbf{W}_{\text {opt-fit }}$ was $41.5 \pm 10.5 \%$ of the sum of squared weights of $\mathbf{W}_{\text {ret }}(34.3,36.6$, and $53.5 \%$, respectively, for three datasets). For comparison, the receptive fields obtained with a randomly selected orthogonal matrix $\mathbf{P}$ (Fig. $2 d$ ) differ substantially from the measured receptive fields, with squared errors of $193.8 \pm 2.4 \%$ (mean \pm SD across three datasets, 100 samples each).

\section{Projective field organization}

Given the non-uniqueness of optimal receptive field structure (above), an incisive test of efficiency would ideally focus on an aspect of retinal circuitry that is both necessary and sufficient for optimality. We find that such unique predictions of efficient coding are given in terms of weights on the signals flowing from a 
(a)
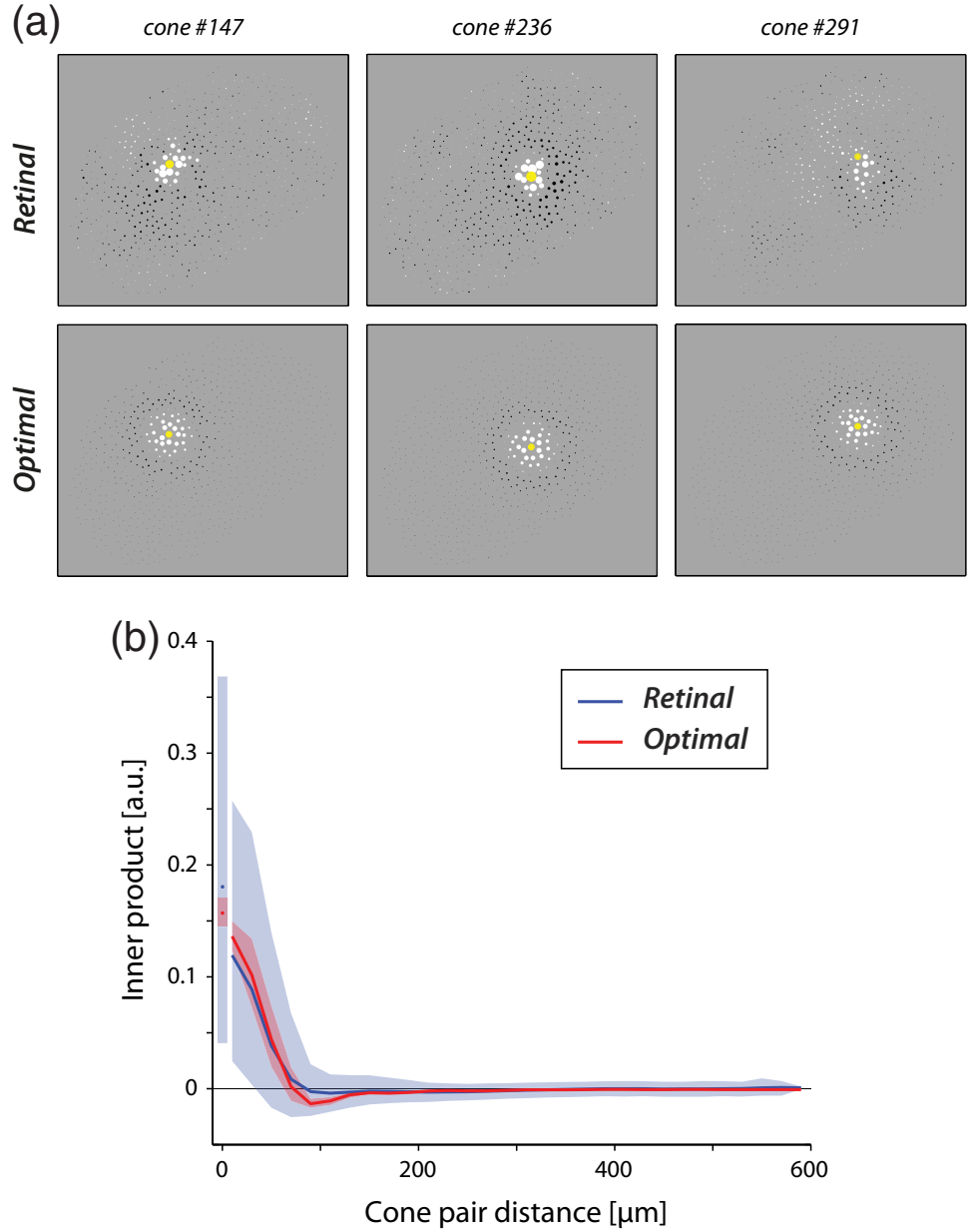

Figure 3. Comparison of cone PF spatial structure to theoretical predictions. $\boldsymbol{a}$, Each panel shows the inner product of the PF of a single cone (yellow) with the PFs of all other cones. The diameter of each circle indicates the magnitude of the inner product; the color indicates the sign (black, negative; white, positive). Top panels show inner products for three cones from the retina; bottom panels show predictions of efficient coding theory for those cones. $\boldsymbol{b}$, The PF inner product as a function of distance between cones. Solid lines indicate average values; shaded regions indicate the 5 th to 95 th percentile range. Values at zero separation indicate squared norms of individual PFs. The gap at small separations reflects the minimum separation between cones in the lattice.

\section{Redundancy}

Is efficient coding consistent with the redundancy observed previously in RGCs (Meister et al., 1995; Puchalla et al., 2005; Schneidman et al., 2006; Shlens et al., 2006; Ala-Laurila et al., 2011)? Because redundancy means that a portion of the information transmitted by one neuron is also transmitted by others (Gawne and Richmond, 1993; Brenner et al., 2000; Machens et al., 2001; Schneidman et al., 2003; Latham and Nirenberg, 2005), one might intuitively expect that a redundant code must be inefficient. Indeed, redundancy reduction has often been stated as an objective synonymous with efficient coding, and, in some special cases, this is correct (Barlow, 1961; Atick and Redlich, 1990; Atick et al., 1990; van Hateren, 1992b; Bell and Sejnowski, 1997). In other cases, however, redundancy can serve to overcome the deleterious effects of noise, improving information transmission (Atick and Redlich, 1990; Atick et al., 1990; van Hateren, 1992b; Barlow, 2001; Zhaoping, 2006; Doi and Lewicki, 2007; Borghuis et al., 2008; Tkacik et al., 2010). This raises the possibility that the redundancy found in the retina is consistent with efficient coding.

As a measure of redundancy, we estimated the fraction of the spatial information conveyed by a single RGC that is also conveyed by other RGCs of the same type (see Materials and Methods). The redundancy associated with the connectivity in the retina $\left(\mathbf{W}_{\text {ret }}\right)$ was $28.7 \pm 7.8 \%$ (mean \pm SD for each cell type, three datasets), whereas the redundancy associated with efficient coding $\left(\mathbf{W}_{\text {opt-fit }}\right)$ was $26.3 \pm$ $11.5 \%$. Although substantial, both of these were much lower than the $86.5 \pm$

particular cone to all the RGCs (across all RGC types), which we refer to as the projective field (PF) of the cone (Lehky and Sejnowski, 1988). More specifically, the unique spatial predictions of efficient coding are the squared magnitudes of the PF of each cone (i.e., strength of the diverging signal) and the similarity between the PFs of different cones (i.e., spatial overlap in their projections to RGCs) (see Materials and Methods).

The complete connectivity maps obtained in the physiological data (Field et al., 2010) provide the first opportunity to compare the spatial structure of cone PFs to the predictions of efficient coding. Figure $3 a$ shows the inner products of the PF of one cone with the PFs of other cones, computed for retinal $\left(\mathbf{W}_{\text {ret }}\right)$ and optimal $\left(\mathbf{W}_{\text {opt }}\right)$ connectivity matrices. In both cases, the inner products assume high positive values for nearby cones (indicating similarity of PFs), smaller negative values for surrounding cones (dissimilarity), and near-zero values for more distant cones. Figure $3 b$ shows that this trend holds for all cone pairs: although the values obtained from the physiological data are significantly more variable than for the optimal solution, the average $\mathrm{PF}$ inner products as a function of distance are consistent with the theory.
$3.7 \%$ redundancy in the cone lattice, analogous to the previous findings of redundancy reduction in the successive stages of auditory sensory systems (Chechik et al., 2006). Also, consistent with previous reports of correlated activity in the retina (Mastronarde, 1989; Meister et al., 1995; Puchalla et al., 2005; Shlens et al., 2006; Ala-Laurila et al., 2011), redundancy between pairs of neighboring cells of the same type was high (up to $30 \%$ ) and declined with distance, for both the retina and the efficient coding model (Fig. 4). In summary, we found that the degree and spatial organization of redundancy in the retina closely matched the predictions of efficient coding.

\section{Discussion}

A detailed test of the efficiency of spatial information coding in the retina was made possible by two advances. First, we developed a tractable model that allowed the computation and optimization of transmitted information in inhomogeneous neural circuits, with multiple constraints on biological resources. Second, we made use of new experimental data and analyses that allow determination of complete functional connectivity between populations of cones and RGCs. The combination of these approaches 
yielded several new findings. First, under these resource constraints, we find that the retina transmits $\sim 80 \%$ of the maximally achievable spatial information about natural images. Second, the functional connectivity between cones and RGCs exhibits unique spatial structure, as captured by PF inner products, consistent with coding efficiency. Finally, the redundancy of spatial information encoded by RGCs has the degree and spatial organization expected from an efficient code.

Previous work has shown that behavioral measurements of visual sensitivity in humans exhibit a bandpass spatial characteristic and changes with light level that are broadly consistent with efficient coding theory (Atick and Redlich, 1990, 1992; van Hateren, 1992b, 1993). Although this result was interpreted in terms of the prototypical centersurround receptive field structure of RGCs, it provided no means to directly compare with physiological measurements. In addition, the theoretical formulation assumed a homogeneous population of rotationally symmetric receptive fields, laid out on a uniform lattice, and equal in number to the cones. In contrast, our formulation (Doi et al., 2010) incorporates much of the variability and irregularity observed in real retinas, including the mismatch in sizes of the populations of cones and RGCs. The use of response variance as a constraint to account for the metabolic cost of spike generation may be found in several previous studies (Atick and Redlich, 1990, 1992; Atick et al., 1990; van Hateren, 1992b, 1993; Ruderman, 1994; Doi and Lewicki, 2007). However, we included an additional constraint on total squared strength of connectivity, reflecting the cost of synaptic maintenance and transmission; this has a significant effect on shaping the solution (Doi et al., 2010). [We chose the L2 norm constraint for connectivity weights because of its analytical tractability (Campa et al., 1995), although the L1 norm constraint may be a more natural choice (Vincent and Baddeley, 2003; Vincent et al., 2005).] Incorporating these constraints, as well as the measured organization of the cone lattice, made it possible to derive and test the theoretical predictions of efficient coding directly in individual retinas.

The present work reveals two novel aspects of efficient coding. First, the theory shows that the necessary and sufficient empirical predictions of efficient coding relate to PF structure rather than receptive field structure. Traditional measurement approaches do not reveal PFs, but the physiological measurement of complete functional circuitry presented here made it possible to test this key theoretical prediction directly. Second, the significant spatial redundancy found among RGCs $(\sim 30 \%)$ is consistent with the predictions of efficient coding (cf. Puchalla et al., 2005; Ala-Laurila et al., 2011). Although previous theoretical work has shown that efficient coding can lead to redundant representations (Atick and Redlich, 1990; Atick et al., 1990; van Hateren, 1992b; Barlow, 2001; Zhaoping, 2006; Doi and Lewicki, 2007; Borghuis et al., 2008; Tkacik et al., 2010) and experimental work has shown that the retinal signals are redundant (Meister et al., 1995; Puchalla et al., 2005; Schneidman et al., 2006; Shlens et al., 2006; Ala-Laurila et al., 2011), the results presented here provide the first direct test of the consistency between these theoretical predictions and experimental results.

The high degree of efficiency exhibited by the retina is presumably achieved through a combination of genetic, developmental, and
Retinal

Optimal (best-fit)

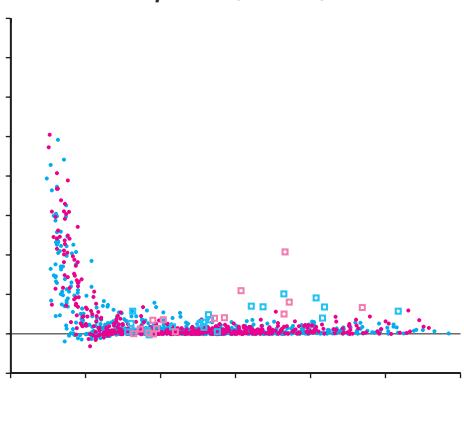

$\begin{array}{cc}200 & 400 \\ R G C & \text { pair distance }[\mu \mathrm{m}]\end{array}$

(1)

Figure 4. Comparison of RGC spatial redundancy to theoretical predictions. Each panel shows the spatial redundancy between pairs of RGCs as a function of the distance between them. Left panels shows results obtained from the measured retinal connectivity, $\mathbf{W}_{\text {ret }}$. Right panel shows results obtained from the optimal connectivity closest to the data, $\mathbf{W}_{\text {opt-fit }}$. Vertical axis shows the fraction of stimulus information conveyed by one cell that is captured by the other; a completely redundant cell pair would exhibit redundancy of 1. Each symbol corresponds to a pair of ON (magenta) or OFF (cyan), Parasol (square) or Midget (dot) RGCs.

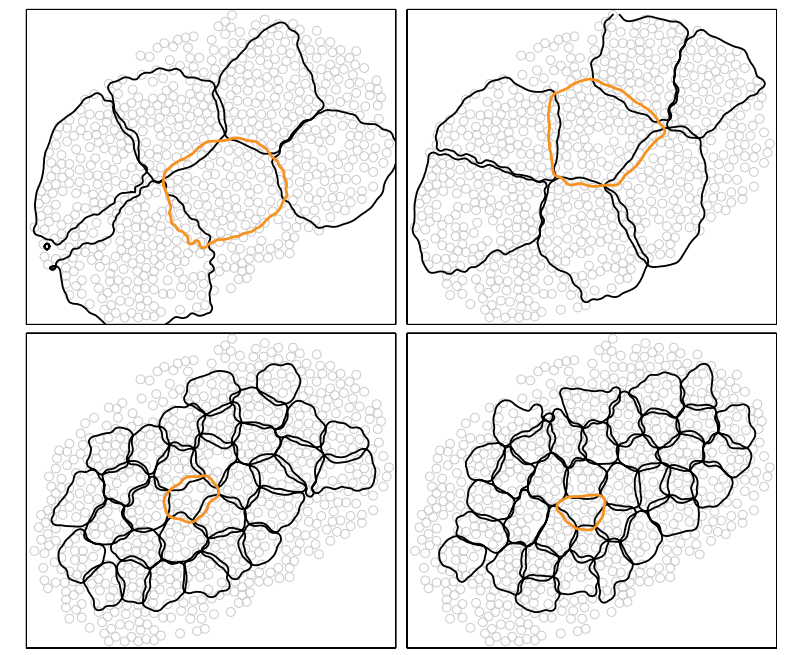

Figure 5. Receptive fields obtained from simulations of the developmental model. Panels show $30 \%$ maximum contours of receptive fields of each cell type (compare to Figure $2 b$ ).

homeostatic mechanisms. It seems unlikely that such mechanisms could be orchestrated to directly optimize information transmission, as we have done in optimizing our model. However, it is natural to ask whether a simpler and more plausible set of constraints might provide a sufficient proxy. Toward this end, a "developmental" model was considered (see Materials and Methods) based on three constraints: (1) the response variances should be constant across RGCs; (2) the PFs of cones to a given RGC type should have fixed magnitude; and (3) long-distance connections between cones and RGCs are prohibited. All three can be plausibly optimized using local learning rules, and all three constraints are consistent with the optimally efficient solution, as well as the regular and uniform arrangement of retinal circuitry. Simulations of this model lead to receptive field structure and organization (Fig. 5), coding efficiency (82.4\%), and redundancy $(26.1 \pm 11.0 \%)$, similar to those observed in the data. We conclude that the retina could, in principle, achieve efficient information transmission and the associated redundancy using simple developmental mechanisms.

Several significant limitations in the analysis of efficiency could be addressed in future work. First, our model does not account for temporal properties of neural response or temporal structure in natural scenes. Inclusion of temporal domain information might help to explain the existence of multiple types of 
RGCs (van Hateren, 1992a; Dong and Atick, 1995). Second, the theory was made tractable by assumptions of linear processing, additive Gaussian noise, and Gaussian signal statistics. All of these assumptions are contradicted to some degree by empirical findings, but substantial advances in analytical methods will be required to incorporate them into the theory (but see Borghuis et al., 2008; Ratliff et al., 2010; Karklin and Simoncelli, 2011; Rahnama Rad and Paninski, 2011; Pitkow and Meister, 2012). Finally, the predictions of efficient coding were tested only in the retina, a neural circuit with unique experimental accessibility that makes high-resolution and complete measurements possible. However, the theory is general and will undoubtedly be investigated in other neural structures as advances in measurement technology permit.

\section{References}

Ala-Laurila P, Greschner M, Chichilnisky EJ, Rieke F (2011) Cone photoreceptor contributions to noise and correlations in the retinal output. Nat Neurosci 14:1309-1316.

Atick JJ, Redlich AN (1990) Towards a theory of early visual processing. Neural Comput 2:308-320.

Atick JJ, Redlich AN (1992) What does the retina know about natural scenes? Neural Comput 4:196-210.

Atick JJ, Li Z, Redlich AN (1990) Color coding and its interaction with spatiotemporal processing in the retina. Princeton: Institute for Advanced Study, IASSNS-HEP-90/75.

Attneave F (1954) Some informational aspects of visual perception. Psychol Rev 61:183-193.

Baddeley R, Abbott LF, Booth MJA, Sengpiel F, Freeman T, Wakeman EA, Rolls ET (1997) Responses of neurons in primary and inferior temporal visual cortices to natural scenes. Proc Biol Sci 264:1775-1783.

Balasubramanian V, Berry MJ 2nd (2002) A test of metabolically efficient coding in the retina. Network 13:531-552.

Balasubramanian V, Sterling P (2009) Receptive fields and functional architecture in the retina. J Physiol 587:2753-2767.

Barlow HB (1961) Possible principles underlying the transformation of sensory messages. In: Sensory communication (Rosenblith WA, ed), pp 217 234. Cambridge, MA: Massachusetts Institute of Technology.

Barlow H (2001) Redundancy reduction revisited. Network 12:241-253.

Baylor DA, Nunn BJ, Schnapf JL (1987) Spectral sensitivity of cones of the monkey Macaca fascicularis. J Physiol 390:145-160.

Bell AJ, Sejnowski TJ (1997) The independent components of natural scenes are edge filters. Vision Res 37:3327-3338.

Bialek W, Rieke F, de Ruyter van Steveninck RR, Warland D (1991) Reading a neural code. Science 252:1854-1857.

Bialek W, de Ruyter van Steveninck RR, Tishby N (2006) Efficient representation as a design principle for neural coding and computation. IEEE International Symposium on Information Theory, Seattle, WA, July 9-14.

Borghuis BG, Ratliff CP, Smith RG, Sterling P, Balasubramanian V (2008) Design of a neuronal array. J Neurosci 28:3178-3189.

Brenner N, Strong SP, Koberle R, Bialek W, de Ruyter van Steveninck RR (2000) Synergy in a neural code. Neural Comput 12:1531-1552.

Campa A, Giudice PD, Parga N, Nadal JP (1995) Maximization of mutual information in a linear noisy network: a detailed study. Network 6:449468.

Chechik G, Anderson MJ, Bar-Yosef O, Young ED, Tishby N, Nelken I (2006) Reduction of information redundancy in the ascending auditory pathway. Neuron 51:359-368.

Chklovskii DB, Schikorski T, Stevens CF (2002) Wiring optimization in cortical circuits. Neuron 34:341-347.

Chong EKP, Zak SH (2001) An introduction to optimization, Ed 2. New York: Wiley.

Cover TM, Thomas JA (2006) Elements of information theory, Ed 2. New York: Wiley.

Dan Y, Atick JJ, Reid RC (1996) Efficient coding of natural scenes in the lateral geniculate nucleus: experimental test of a computational theory. J Neurosci 16:3351-3362.

De Valois RL, Morgan H, Snodderly DM (1974) Psychophysical studies of monkey vision. III. Spatial luminance contrast sensitivity test of macaque and human observers. Vision Res 14:75-81.

Devries SH, Baylor DA (1997) Mosaic arrangement of ganglion cell receptive fields in rabbit retina. J Neurophysiol 78:2048-2060.

Dhingra NK, Smith RG (2004) Spike generator limits efficiency of information transfer in a retinal ganglion cell. J Neurosci 24:2914-2922.

Doi E, Inui T, Lee TW, Wachtler T, Sejnowski TJ (2003) Spatiochromatic receptive field properties derived from information-theoretic analyses of cone mosaic responses to natural scenes. Neural Comput 15:397-417.

Doi E, Lewicki MS (2007) A theory of retinal population coding. In: Advances in neural information processing systems, Vol 19, pp 353-360. Cambridge, MA: Massachusetts Institute of Technology.

Doi E, Paninski L, Simoncelli EP (2010) Diversity of efficient coding solutions for a population of noisy linear neurons. Presented at the Annual Meeting of Computational and Systems Neuroscience. Salt Lake City, UT, February.

Dong DW, Atick JJ (1995) Temporal decorrelation: a theory of lagged and nonlagged responses in the lateral geniculate nucleus. Network 6:159-178.

Field GD, Gauthier JL, Sher A, Greschner M, Machado TA, Jepson LH, Shlens J, Gunning DE, Mathieson K, Dabrowski W, Paninski L, Litke AM, Chichilnisky EJ (2010) Functional connectivity in the retina at the resolution of photoreceptors. Nature 467:673-677.

Gauthier JL, Field GD, Sher A, Greschner M, Shlens J, Litke AM, Chichilnisky EJ (2009) Receptive fields in primate retina are coordinated to sample visual space more uniformly. PLoS Biol 7:e1000063.

Gawne TJ, Richmond BJ (1993) How independent are the messages carried by adjacent inferior temporal cortical neurons? J Neurosci 13:2758-2771.

Gower JC, Dijksterhuis GB (2004) Procrustes problems. Oxford, UK: Oxford UP.

Graham DJ, Chandler DM, Field DJ (2006) Can the theory of "whitening" explain the center-surround properties of retinal ganglion cell receptive fields? Vision Res 46:2901-2913.

Haft M, van Hemmen JL (1998) Theory and implementation of infomax filters for the retina. Network 9:39-71.

Karklin Y, Simoncelli EP (2011) Efficient coding of natural images with a population of noisy linear-nonlinear neurons. In: Advances in neural information processing systems (Shawe-Taylor J, Zemel R, Bartlett P, Pereira F, Weinberger K, eds), Vol 24, pp 999-1007. Cambridge, MA: Massachusetts Institute of Technology

Kelly DH (1972) Adaptation effects on spatio-temporal sine-wave thresholds. Vision Res 12:89-101.

Koch K, McLean J, Berry M, Sterling P, Balasubramanian V, Freed MA (2004) Efficiency of information transmission by retinal ganglion cells. Curr Biol 14:1523-1530.

Latham PE, Nirenberg S (2005) Synergy, redundancy, and independence in population codes, revisited. J Neurosci 25:5195-5206.

Laughlin S (1981) A simple coding procedure enhances a neuron's information capacity. Z Naturforsch 36:910-912.

Laughlin SB (2001) Energy as a constraint on the coding and processing of sensory information. Curr Opin Neurobiol 11:475-480.

Lehky SR, Sejnowski TJ (1988) Network model of shape-from-shading: neural function arises from both receptive and projective fields. Nature 333:452-454.

Linsker R (1989) An application of the principle of maximum information preservation to linear systems. In: Advances in neural information processing systems, Vol 1, pp 186-194. Cambridge, MA: Massachusetts Institute of Technology.

Liu YS, Stevens CF, Sharpee TO (2009) Predictable irregularities in retinal receptive fields. Proc Natl Acad Sci U S A 106:16499-16504.

Machens CK, Stemmler MB, Prinz P, Krahe R, Ronacher B, Herz AV (2001) Representation of acoustic communication signals by insect auditory receptor neurons. J Neurosci 21:3215-3227.

Masland RH (2001) The fundamental plan of the retina. Nature neuroscience 4:877-886.

Mastronarde DN (1989) Correlated firing of retinal ganglion cells. Trends Neurosci 12:75-80.

Meister M, Lagnado L, Baylor DA (1995) Concerted signaling by retinal ganglion cells. Science 270:1207-1210.

Navarro R, Artal P, Williams DR (1993) Modulation transfer of the human eye as a function of retinal eccentricity. J Opt Soc Am A 10:201-212.

Olshausen BA, Field DJ (1996) Emergence of simple-cell receptive field 
properties by learning a sparse code for natural images. Nature 381:607-609.

Pitkow X, Meister M (2012) Decorrelation and efficient coding by retinal ganglion cells. Nat Neurosci 15:628-635.

Puchalla JL, Schneidman E, Harris RA, Berry MJ (2005) Redundancy in the population code of the retina. Neuron 46:493-504.

Rahnama Rad K, Paninski L (2011) Information rates and optimal decoding in large neural populations. In: Advances in neural information processing systems (Shawe-Taylor J, Zemel R, Bartlett P, Pereira F, Weinberger K, eds), Vol 24, pp 846-854. Cambridge, MA: Massachusetts Institute of Technology.

Ratliff CP, Borghuis BG, Kao YH, Sterling P, Balasubramanian V (2010) Retina is structured to process an excess of darkness in natural scenes. Proc Natl Acad Sci U S A 107:17368-17373.

Rieke F, Bodnar DA, Bialek W (1995) Naturalistic stimuli increase the rate and efficiency of information transmision by primary auditory afferents. Proc Biol Sci 262:259-265.

Rieke F, Warland DK, de Ruyter van Steveninck RR, Bialek W (1997) Spikes: exploring the neural code. Cambridge, MA: Massachusetts Institute of Technology.

Ruderman DL (1994) Designing receptive fields for highest fidelity. Network 5:147-155.

Schneidman E, Bialek W, Berry MJ 2nd (2003) Synergy, redundancy, and independence in population codes. J Neurosci 23:11539-11553.

Schneidman E, Berry MJ 2nd, Segev R, Bialek W (2006) Weak pairwise correlations imply strongly correlated network states in a neural population. Nature 440:1007-1012.
Schwartz O, Simoncelli EP (2001) Natural signal statistics and sensory gain control. Nat Neurosci 4:819-825.

Shlens J, Field GD, Gauthier JL, Grivich MI, Petrusca D, Sher A, Litke AM, Chichilnisky EJ (2006) The structure of multi-neuron firing patterns in primate retina. J Neurosci 26:8254-8266.

Smith EC, Lewicki MS (2006) Efficient auditory coding. Nature 439: 978-982.

Srinivasan MV, Laughlin SB, Dubs A (1982) Predictive coding: a fresh view of inhibition in the retina. Proc Biol Sci 216:427-459.

Strang G (2005) Linear algebra and its applications, Ed 4. Stamford, CT: Brooks Cole.

Tkacik G, Prentice JS, Balasubramanian V, Schneidman E (2010) Optimal population coding by noisy spiking neurons. Proc Natl Acad Sci U S A 107:14419-14424.

van Hateren JH (1993) Spatiotemporal contrast sensitivity of early vision. Vision Res 33:257-267.

van Hateren JH (1992a) Theoretical predictions of spatiotemporal receptive fields of fly LMCs, and experimental validation. J Comp Physiol A Neuroethol Sens Neural Behav Physiol 171:157-170.

van Hateren JH (1992b) A theory of maximizing sensory information. Biol Cybern 68:23-29.

Vincent BT, Baddeley RJ (2003) Synaptic energy efficiency in retinal processing. Vision Res 43:1283-1290.

Vincent BT, Baddeley RJ, Troscianko T, Gilchrist ID (2005) Is the early visual system optimised to be energy efficient? Network 16:175-190.

Zhaoping L (2006) Theoretical understanding of the early visual processes by data compression and data selection. Network 17:301-334. 